\title{
The Use of Pistacia Lentiscus Chia Resin versus Omeprazole in Protecting Male Rats Peptic Mucosa against Cold Restraint Stress
}

\author{
Despoina Kakagia1* ${ }^{*}$, Apostolos Papalois², Maria Lambropoulou³ ${ }^{3}$ Fotini Papachristou1, \\ Gregory Trypsiannis ${ }^{4}$, Constantinos Anagnostopoulos ${ }^{5}$, Mike Pitiakoudis ${ }^{1}$, Alexandra \\ Tsaroucha ${ }^{1}$ \\ 1 Democritus University of Thrace Faculty of Medicine Alexandroupolis, Greece \\ 2 Elpen Research Centre, Elpen Pharmaceuticals, Greece \\ 3 Department of Histology- Embryology, Democritus University of Thrace, Faculty of Medicine, Alexandroupolis, \\ Greece \\ ${ }^{4}$ Department of Medical Statistics, Democritus University of Thrace, Faculty of Medicine, Alexandropoulis, Greece \\ 5 Department of Biochemistry, Democritus University of Thrace, Faculty of Medicine, Alexandroupolis, Greece
}

\begin{abstract}
Introduction: Peptic mucosal damage induced by acute stress is a serious cause of morbidity and mortality in critically ill patients. The study aimed to investigate the protective, antioxidant and anti-inflammatory effects of pretreatment with Chios mastic gum (CMG), a traditionally consumed herbal resin naturally deriving from the trunk of Pistacia Lentiscus var. Chia compared to Omeprazole, a standard medication used in the prevention and treatment of gastritis, against the effects of cold restraint stress (CRS) in rat gastric and colonic mucosa. Methods: Twenty-one male Wistar rats were randomly assigned to three groups: Control (C), Omeprazole (O), and CMG (M), according to the pre-treatment regime, and were subjected to $\mathrm{CRS}$ at $4^{\circ} \mathrm{C}$ for 3 hours. The gastric and colonic mucosal lesions were histologically assessed. ELISA measured blood concentrations of TNF- $\alpha$, IL-1 $\beta$, peroxidase, superoxide dismutase (SOD) and total antioxidant capacity (TEAC). Results: In both groups, $\mathrm{O}$ and $\mathrm{M}$, gastric mucosal hyperemia, haemorrhagic infiltration and mucosal oedema, as well as colonic mucosal hyperaemia and haemorrhagic infiltration were significantly reduced compared to the controls $(p<0.05)$. No significant differences were observed between Groups $O$ and M. TNF- $\alpha$ levels were significantly lower in group M compared to Group O $(p=0.013)$. IL- $1 \beta$ levels were significantly depressed in groups $M$ and $O$ compared to control $(p \leq 0.001)$. The activity of both peroxidase and SOD enzymes decreased in group $M$ compared to group $O(p=0.043$ and $p=0.047$ respectively) and the control $(p=0.018$ and $p<0.001$ respectively). Conclusions: The natural Chios mastic gum is a promising nutritional supplement with protective properties to the peptic mucosa against CRS, exerting anti-inflammatory and antioxidant effects.
\end{abstract}

Keywords: cold restraint stress, colitis, gastric ulcer, oxidative stress, Pistacia Lentiscus Chia, Omeprazole

Received: 11 February 2020 / Accepted: 14 April 2020

\section{INTRODUCTION}

Stress gastritis, and less often stress colitis, occurs in critically ill patients following, amongst other matters, trauma, sepsis, shock, multi-organ failure or those undergoing prolonged surgical treatment [1-4]. Lesions of the gastric mucosa may be focal or diffuse, superficial or potentially resulting in ulceration or gastric haemorrhage, which is associated with significant morbidity and mortality.

The predominant theory of the pathogenesis of gastric mucosal damage caused by stress was, until recent- ly, the destruction of the gastric mucosal barrier due to ischemia, which renders the stomach vulnerable to gastric fluids [1-3].

Experimental immobilisation models, involving either exposure to cold [4-8], or water immersion [928 ], are well documented as inducing gastric mucosal lesions in rodents. It has been found that each model produces a stimulus that rapidly activates the sympathetic adrenomedullary system, reduces blood flow to the gastric mucosa causing local hypoxia and ischemia, epithelial necrosis and haemorrhagic gastric mucosal corrosion [5-28]. 
Cold restraint stress (CRS) has been found to induce gastric lesions which are associated with decreased or normal levels of acid secretions [27, 29]. Administration of antacids to neutralise secreted acid does not prevent stress ulcer [30], suggesting that factors other than gastric acid are involved in ulcer formation. Reactive oxygen species (ROS) such as $\cdot \mathrm{O}_{2}{ }^{-}$and $\cdot \mathrm{OH}$ are now considered to be major causative factors for mucosal lesions through oxidative damage [21, 22, 27, 31-33].

Colitis, due to stress, is less frequently found in severely ill patients and has not been clinically investigated because colorectal lesions are often due to drugdamage or disorders of the colonic flora caused by medication.

It has been reported that in experimental rodent models, stress caused by immobilisation contributes to the development of colitis [4]. To our knowledge, none of the CRS experimental models has been used to study stress-induced colonic mucosal changes in rodents [4, $5,29]$.

Chios mastic gum $(\mathrm{CMG})$ is a resin naturally produced exclusively in the southern region of the Greek island of Chios, in the Northern Aegean Sea, by prickling the trunk of the shrub Pistacia lentiscus var. Chia. This traditional gum is very popular as a nutritional ingredient, due to its distinct essence, the Eastern Mediterranean and Arab countries. Its beneficial health effects, mainly for gastrointestinal disorders, have been known for more than 2500 years, as documented by Dioscurides and Galen.

Previous studies showed that CMG possesses antiulcer activities [2, 3], while antibacterial, antioxidant, and chemopreventive properties of CMG have also been documented [22, 34-38]. Terpenoids are the active compounds of CMG exerting an anti-inflammatory as well as antioxidant effects [36, 37].

Chios mastic gum is effective in the treatment of drug-induced gastroduodenal ulcers and colitis in rats $[22,36,37]$, but without any effect as an in vivo monotherapy against Helicobacter pylori [38, 39].

Omeprazole has been a standard prevention and treatment medication for peptic ulcer disease since 1989. Indications include gastroesophageal reflux disease, Zollinger-Elisson syndrome, as well as eosinophilic gastritis. It is also used to prevent upper gastrointestinal bleeding in people who are at high risk but has been associated with certain adverse effects and drug interactions $[40,41]$.
Given that mastic is a natural product of widespread use in many countries, its potential protective effect on the gastric and colonic mucosa may render it a key target for the pharmacological management of critically ill patients. The prevention of stress-induced changes of the gastric and colonic mucosa via nutritional interventions may significantly reduce morbidity and mortality of critically ill patients.

The present study aimed to investigate the protective, antioxidant and anti-inflammatory action of CMG pre-treatment versus Omeprazole, on the CRS effects induced in the rat gastric and colonic mucosa.

The null hypothesis is that CMG has no protective action on the rat peptic mucosa against CRS.

\section{MATERIALS AND METHODS}

Twenty-one male Wistar rats, aged 12-14 months and weighing 320-380 g, were used in this study [42].

The animals were housed in wire cages under standard laboratory conditions. They had been acclimated for two weeks before the experiment in the laboratory. The conditions were $12 \mathrm{~h}$ light-dark cycles, $22-25^{\circ} \mathrm{C}$ room temperature, and 55-58\% humidity. The animals were freely fed with a standard laboratory usual pellet diet and water intake.

All experimental procedures in this study were conducted under the Regional State License 1635/1503-2016 and following the European guidelines of Directive $86 / 609 /$ EEC on the protection and welfare of animals used for experimental and other scientific purposes.

The sample size was determined by power analysis.

The animals were randomly assigned to the following three groups, each of seven animals: Control (C), Omeprazole $(\mathrm{O})$, and $\mathrm{CMG}(\mathrm{M})$.

\section{Preparation of administered substances}

The substances used were:

1.Omeprazole suspension (Omeprazole \& SyrSpend ${ }^{\circ}$ SF Alka Convenience Pack 2 mg/mL, Fargon Hellas-Kertus ICSA, Trikala, Hellas)

2.Chios Mastic natural resin ground in microparticulate form (Chios Mastic Producers Association, Chios, Greece), chemically analysed and suspended in water by shaking.

3. Control group animals received tap water in place of the active agents. 
All substances were prepared immediately before use and administered via an orogastric tube.

\section{The experimental protocol}

Two days before the induced stress, the following was administered to the animals, once a day by orogastric tube.

Group C, additional water $10 \mathrm{~mL}$; 26].

Group O, omeprazole $20 \mathrm{mg} / \mathrm{kg}$ in water;[21].

Group M, Chios mastic gum powder $500 \mathrm{mg}$ in micro-particulate form suspended in water [22,23]. On the day before the experiment, in order to avoid coprophagy, the animals were placed in wire-bottomed cages and were deprived of food for 24 hours, but had free access to tap water and received the pre-treatment as mentioned above regimen.

On the day of the experiment, the animals were transferred to Bollmann cages, immobilised and kept at a temperature of $4^{\circ} \mathrm{C}$ for three hours [8].

At the end of the experiment, the animals were anesthetized with ketamine $100 \mathrm{mg} / \mathrm{kg}$ BW (Ketamin, IFET , Athens, Greece) and xylazine $20 \mathrm{mg} / \mathrm{kg} \mathrm{BW}$ (Xylamed, Bimeda INC, Oakbrook Terrace, IL, USA). The abdomen of each animal was then opened and 1,5-2,5 $\mathrm{mL}$ of blood was obtained from the abdominal aorta for measurement of oxidative stress and inflammation markers by ELISA [12].

The entire stomach and ascending colon were then removed for histological examination. All blood and tissue samples were appropriately encoded in order to ensure blind assessment. The animals were euthanized by an intracardiac infusion of $0.6 \mathrm{mg} / \mathrm{kg}$ BW pentobarbital sodium BP20\% (Dolethal, Vetoquinol Ltd, UK)

Determination of serum antioxidant and anti-inflammatory parameters

The oxidative status was evaluated by determination of the oxidative stress markers peroxidase, total antioxidant capacity in Trolox equivalents (TEAC), and superoxide dismutase (SOD). The anti-inflammatory properties were evaluated by measurement of tumour necrosis factor-alpha (TNF- $\alpha$ ) and interleukin-1 beta (IL-1 $\beta$ ). The concentration of each marker in serum samples was measured by enzyme-linked immunosorbent assay (ELISA).

The Superoxide Dismutase Activity (SOD) researchuse-only kit (Cayman Chemical Company, Ann Arbor, MI, USA) was used to determine serum superoxide dismutase activity $(\mathrm{U} / \mathrm{mL})$.
The assay was performed according to the manufacturer's instructions. Rat serum samples were diluted 1:5 with sample buffer, and the absorbance was measured at $450 \mathrm{~nm}$.

TNF- $\alpha$ and IL- $1 \beta$ serum levels $(\mathrm{pg} / \mathrm{mL})$ were determined by ELISA according to the manufacturer's instructions (rat TNF-alpha ELISA kit and rat IL-1 beta ELISA kit, Raybiotech, Norcross, GA, USA). Rat serum samples were diluted 1:2 with assay diluent $A$. The absorbance was measured at $450 \mathrm{~nm}$.

Antioxidant and peroxide assay kits (Sigma-Aldrich, St. Louis, MO, USA) were employed to determine the total antioxidant capacity and peroxide concentration in serum samples. Both assays were performed according to the manufacturer's instructions. Total antioxidant capacity ( $\mathrm{mM}$ of Trolox equivalents) was determined in serum samples diluted 1:20 with assay buffer, and the absorbance was measured at $405 \mathrm{~nm}$.

Peroxide concentration $\left(\mu \mathrm{M} \mathrm{H}_{2} \mathrm{O}_{2}\right)$ was determined in serum samples diluted 1:2 with ultrapure water (Cayman chemical company, Ann Arbor, MI, USA) and the absorbance was measured at $592 \mathrm{~nm}$.

Absorbance measurements were performed using Expertplus (ASYS GmBH, Austria) microtiter plate reader.

\section{Pathological assessment of tissues}

Immediately after removal, the stomachs were inflated by an injecting one $\mathrm{mL}$ of $2 \%$ formalin then fixed in $2 \%$ formalin for ten minutes before being opened along the greater curvature and rinsed with saline to remove the gastric content and clots, before stabilisation in $10 \%$ formalin solution. Sections, $5 \mu \mathrm{m}$ thick, were cut on a microtome (HIRAX M60, Carl Zeiss, Germany) and stained with haematoxylin and eosin (HE).

Gastric mucosal lesions were studied by dissecting microscopy, and the lesion score was calculated.

The damage scores were categorised as follows:

- A one $\mathrm{cm}$ segment of each histological section was assessed for:

- hyperaemia (score: $0-3$ )

- loss of epithelial cells (EC) (score: 0-3)

- oedema in the upper mucosa (score: $0-3$ )

- haemorrhagic infiltration (score: $0-3$ )

- presence of inflammatory cells (IC) (score: 0-3) $[19,21]$.

- The average score of gastric mucosal lesions in each group of animals was calculated, and the results were statistically analysed. 
- The ascending colon was immersed and rinsed. Prepared colonic mucosal sections were stabilised with $10 \%$ formalin solution; paraffin blocks were formed and stained with HE and Periodic acid-Schiff (PAS). The number of mucin-containing cell-surface cells was quantitated in a region containing ten parallel crypts $[4,43]$.

- The results were expressed as the number of mucuscontaining cup cells per 100 epithelial cells of the colon [4]. Quantitative measurement of colonic mucin was not conducted.

- The colonic mucosal histological damages identified were:

- hyperaemia (score: 0-3) and

- haemorrhagic infiltration (score: $0-3$ )

- The average score of colonic mucosal lesions in each group of animals was calculated, and the results were statistically analysed.

- The same pathologist blindly screened all encoded preparations.

\section{Statistical analysis}

Statistical analysis of the data was performed using SPSS version 19.0 (IBM, Corp.).

The normality of quantitative variables was tested by the Kolmogorov-Smirnov test.

All serum antioxidant and anti-inflammatory parameters were expressed as mean and standard deviation, $[\operatorname{mean}(\mathrm{SD})]$. .

Differences of these parameters between the three groups were assessed by one-way analysis of variance, (ANOVA).

To show the exact differences between the three different experimental groups, multiple comparisons were performed using the Least Significant Difference (LSD) test, without any adjustment of the significance level.

All indices of the pathological assessment of the tissues, hyperaemia, haemorrhagic infiltration, loss of EC, mucosal oedema and presence of IC for the gastric mucosa, and hyperaemia and haemorrhagic infiltration for the colonic mucosa, were expressed as absolute frequencies and percentages (\%) when they were considered as qualitative variables, and as Mean(SD) and Median (min-max) values, when they were considered as quantitative variables.

They were analysed using chi-square test, ANOVA and Kruskal-Wallis test, respectively.

All tests were two-tailed, and the significance level was set at $\alpha=0.05$.

\section{RESULTS}

\section{Chios Mastic Gum Analysis}

The supplied resin was chemically analysed and was found to contain: natural Polymer - cis-1,4 poly- $\beta$ myrcene $(25-30 \% \mathrm{w} / \mathrm{w})$, mastic oil $(2-3 \% \mathrm{w} / \mathrm{w})$, total mastic extract $(70 \% \mathrm{w} / \mathrm{w})$, acid fraction $(38-42 \% \mathrm{w} / \mathrm{w})$ and neutral fraction $(28-32 \% \mathrm{w} / \mathrm{w})$.

\section{Gastric mucosal response to CRS}

Following CRS, rats developed gastric mucosal lesions such as hyperaemia, haemorrhagic infiltration, mucosal oedema, loss of EC and infiltration by IC in all three groups (Figure 1).

The severity of these lesions was affected by the prior administration of either omeprazole or mastic powder.

As shown in Table 1, measurements of hyperaemia, haemorrhagic infiltration, and mucosal oedema were
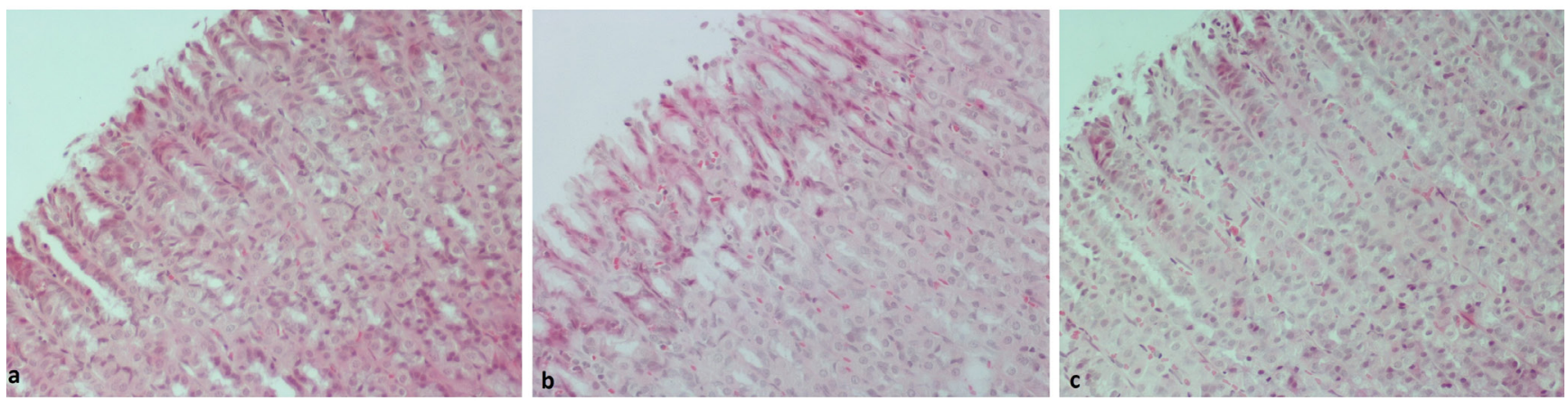

Fig. 1. Gastric mucosa sections. H E x 200. Mucosal changes in: a. Chios mastic group: minimal hyperaemia and oedema, rare inflammatory cells, b. omeprazole group: mild hyperemia and oedema, minor deposits of inflammatory cells c. control: more extensive hyperemia and oedema and deposits of inflammatory cells, moderate hemorrhagic infiltration and pronounced loss of epithelial cells. 
Table 1. Gastric mucosal lesions in the three groups

\begin{tabular}{|c|c|c|c|c|c|c|c|}
\hline & & Groups & & & Multi & e compari & sons \\
\hline & C & 0 & $\mathbf{M}$ & $p$ value & C vs 0 & C vs M & O vs $\mathrm{M}$ \\
\hline Hyperemia & & & & $0.001^{a}$ & & & \\
\hline Negative & $0(0.0)$ & $2(28.6)$ & $1(14.3)$ & & & & \\
\hline Low & $0(0.0)$ & $5(71.4)$ & $6(85.7)$ & & & & \\
\hline Moderate & $6(85.7)$ & $0(0.0)$ & $0(0.0)$ & & & & \\
\hline High & $1(14.3)$ & $0(0.0)$ & $0(0.0)$ & & & & \\
\hline Mean value (SD) & $2.14(0.38)$ & $0.71(0.49)$ & $0.86(0.38)$ & $<0.001^{b}$ & $<0.001^{c}$ & $<0.001^{c}$ & $0.530^{c}$ \\
\hline Median value (min-max) & $2(2-3)$ & $1(0-1)$ & $1(0-1)$ & $<0.001^{d}$ & $0.001^{\mathrm{e}}$ & $0.001^{\mathrm{e}}$ & $0.530^{\mathrm{e}}$ \\
\hline Hemorrhagic infiltration & & & & 0.001 & & & \\
\hline Negative & $0(0.0)$ & $7(100.0)$ & $6(85.7)$ & & & & \\
\hline Low & $4(57.1)$ & $0(0.0)$ & $1(14.3)$ & & & & \\
\hline Moderate & $3(42.9)$ & $0(0.0)$ & $0(0.0)$ & & & & \\
\hline High & $0(0.0)$ & $0(0.0)$ & $0(0.0)$ & & & & \\
\hline Mean value (SD) & $1.43(0.54)$ & $0.0(0.0)$ & $0.14(0.38)$ & $<0.001$ & $<0.001$ & $<0.001$ & 0.489 \\
\hline Median value (min-max) & $1(1-2)$ & 0 & $0(0-1)$ & $<0.001$ & 0.001 & 0.002 & 0.317 \\
\hline Loss EC & & & & 0.084 & & & \\
\hline Negative & $0(0.0)$ & $4(57.1)$ & $3(42.9)$ & & & & \\
\hline Low & $5(71.4)$ & $3(42.9)$ & $4(57.1)$ & & & & \\
\hline Moderate & $2(28.6)$ & $0(0.0)$ & $0(0.0)$ & & & & \\
\hline High & $0(0.0)$ & $0(0.0)$ & $0(0.0)$ & & & & \\
\hline Mean value (SD) & $1.29(0.49)$ & $0.43(0.54)$ & $0.57(0.54)$ & 0.014 & 0.006 & 0.019 & 0.613 \\
\hline edian value (min-max) & $1(1-2)$ & $0(0-1)$ & $1(0-1)$ & 0.026 & 0.015 & 0.030 & 0.606 \\
\hline Oedema & & & & $0.017^{a}$ & & & \\
\hline Negative & $0(0.0)$ & $5(71.4)$ & $4(57.1)$ & & & & \\
\hline Low & $7(100.0)$ & $2(28.6)$ & $3(42.9)$ & & & & \\
\hline Moderate & $0(0.0)$ & $0(0.0)$ & $0(0.0)$ & & & & \\
\hline High & $0(0.0)$ & $0(0.0)$ & $0(0.0)$ & & & & \\
\hline Mean value (SD) & $1.00(0.0)$ & $0.29(0.49)$ & $0.43(0.54)$ & $0.012^{b}$ & $0.005^{c}$ & $0.020^{c}$ & $0.530^{c}$ \\
\hline Median value (min-max) & 1 & $0(0-1)$ & $0(0-1)$ & $0.020^{d}$ & $0.007 \mathrm{e}$ & $0.023^{e}$ & $0.591^{\mathrm{e}}$ \\
\hline Infiltration IC & & & & 0.466 & & & \\
\hline Negative & $3(42.9)$ & $5(71.4)$ & $3(42.9)$ & & & & \\
\hline Low & $4(57.1)$ & $2(28.6)$ & $4(57.1)$ & & & & \\
\hline Moderate & $0(0.0)$ & $0(0.0)$ & $0(0.0)$ & & & & \\
\hline High & $0(0.0)$ & $0(0.0)$ & $0(0.0)$ & & & & \\
\hline Mean value (SD) & $0.57(0.54)$ & $0.29(0.49)$ & $0.57(0.54)$ & 0.507 & 0.317 & 1.000 & 0.317 \\
\hline Median value (min-max) & $1(0-1)$ & $0(0-1)$ & $1(0-1)$ & 0.483 & 0.298 & 1.000 & 0.298 \\
\hline Average histological score, mean (SD) & $6.43(1.13)$ & $1.71(0.95)$ & $2.57(1.62)$ & $<0.001$ & $<0.001$ & $<0.001$ & 0.221 \\
\hline
\end{tabular}

less pronounced in Groups $\mathrm{O}$ and $\mathrm{M}$ compared to Group C (chi-square test ; $\mathrm{p}<0.05)$, (ANOVA; $\mathrm{p}<0.05$ ), ( Kruskal-Wallis test; $\mathrm{p}<0.05$ ), for categorical or continuous variables, respectively (Figure 2). The same applied for the loss of EC. (chi-square test; $\mathrm{p}=0.084$.) There were no statistical differences for any of these parameters, between Groups O and M (LSD test and Mann-Whitney U-test; $\mathrm{p}>0.05$ ).
No statistically significant differences between the three groups were observed regarding the presence of IC. ( $p>0.05$; chi-square test, ANOVA and KruskalWallis test;). Furthermore, the average total histological score was significantly lower in Group O ( $\mathrm{p}<0.001$;LSD test and Group $\mathrm{M}(\mathrm{p}<0.001 ; \mathrm{LSD}$ test) compared to group C. No statistically significant difference was found between Groups $\mathrm{O}$ and $\mathrm{M}$ ( $\mathrm{p}=0.221$; LSD test). 


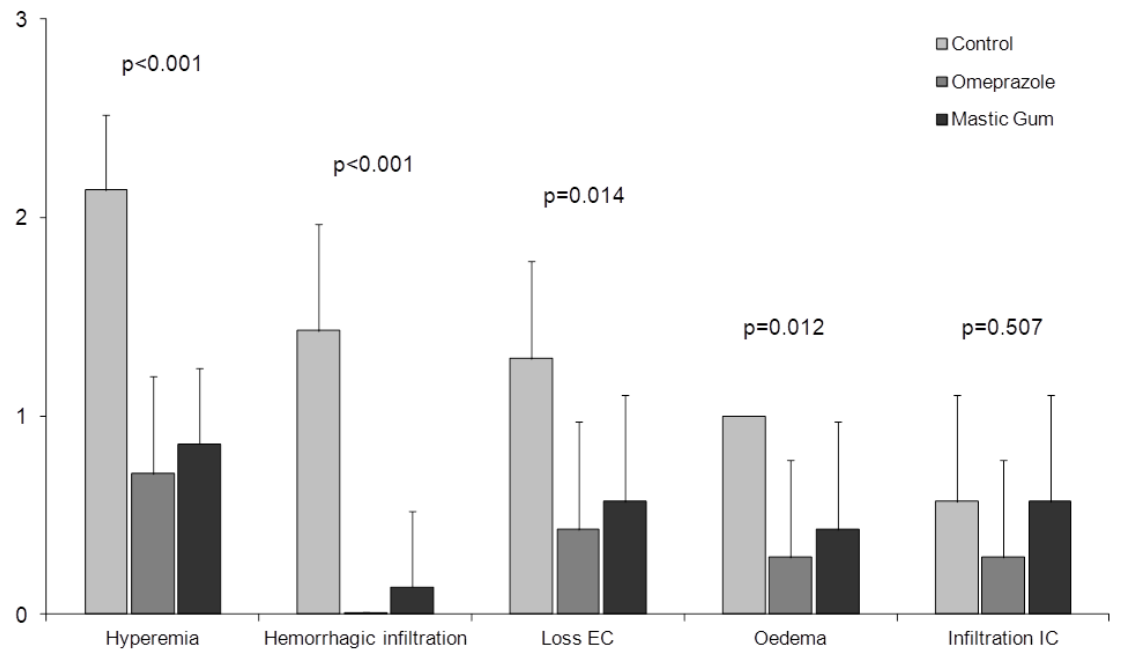

Fig. 2. Graphic for statistic comparison of gastric mucosal lesions between the three groups.

Effects of omeprazole and mastic gum on colonic mucosa

In all three groups of animals, cold restraint of rats at $4^{\circ} \mathrm{C}$ for three hours produced mucosal changes, as histologically observed in sections of the ascending colon,

Mucosal changes such as hyperaemia and haemorrhagic infiltration were observed in examined sections. The response of the colonic mucosa to the stimulus of stress was calculated by the number of mucin-containing Goblet cells (Figure 3) [43].

Measurements of hyperaemia and haemorrhagic infiltration of colonic mucosal lesions were less pronounced in Groups $\mathrm{O}$ and $\mathrm{M}$ compared to Group C. $(\mathrm{p}=0.001$ and $\mathrm{p}=0.002$, respectively; Chi-square test $)$ or $(\mathrm{p}<0.001 ;$ ANOVA $)$ and $(\mathrm{p}<0.001$; Kruskal-Wallis test; $)$ (Table 2 and Figure 4).

Both hyperaemia and haemorrhagic infiltration were similar between Groups $\mathrm{O}$ and M ( $\mathrm{p}>0.05$; LSD test and Mann-Whitney U-test). Furthermore, the average total histological score was significantly lower in Group O
( $p<0.001 ;$ LSD test) and Group $M(p<0.001 ;$ LSD test) compared to Group C. No statistically significant difference was found between Groups $\mathrm{O}$ and $\mathrm{M}(\mathrm{p}=0.119$; LSD test). No statistically significant differences between the three groups were observed concerning the number of colonic Goblet cells ( $p=0.370$;ANOVA) Table 2 .

\section{Antioxidative and anti-inflammatory properties of Omeprazole and mastic resin}

Table 3 contains the results for the comparison of the anti-inflammatory and the oxidative stress markers between the three groups, both altogether and in pairs (Figure 5).

Statistically significant differences of peroxidase levels were observed between the three groups $(p=0.039$; ANOVA).

Post-hoc analysis revealed that $27.2 \%$ lower peroxidase levels were observed in Group M compared to Group C ( $\mathrm{p}=0.018$; LSD test) and by $23.6 \%$ in Group O $(\mathrm{p}=0.043 ; \mathrm{LSD}$ test $)$.
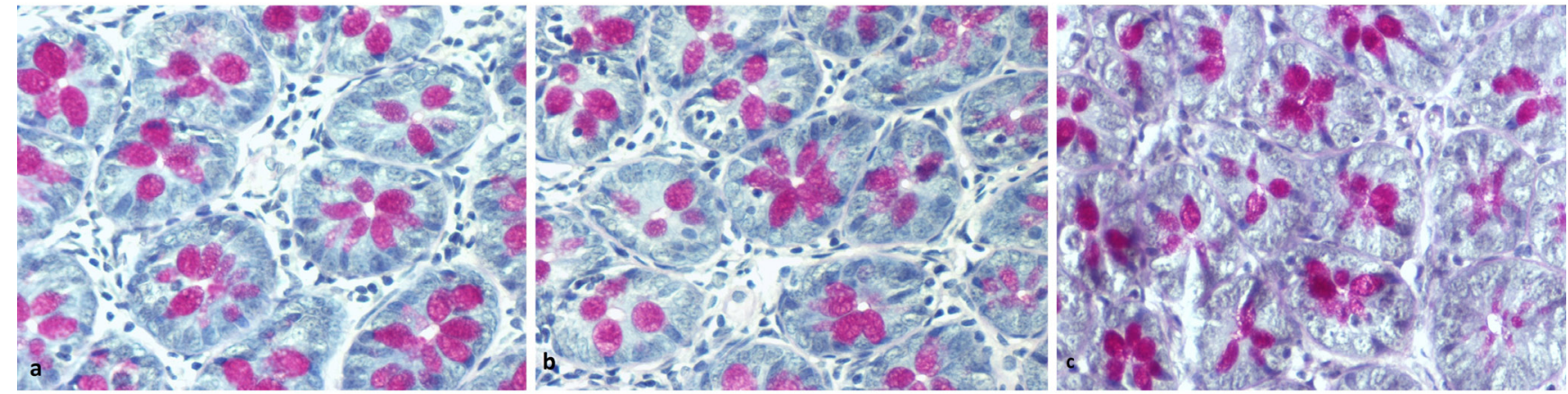

Fig. 3. Mucin containing mucosal Goblet Cells in histologic sections of the ascending colon, presenting as pink flower petals. Periodic acid-Schiff $(P A S) \times$ 200. a. after pre-treatment with CMG, b. after pre-treatment with Omeprazole and c. in the control group. 
Table 2. Mucosal lesions observed in the ascending colon in the three groups

\begin{tabular}{|c|c|c|c|c|c|c|c|}
\hline & \multicolumn{3}{|c|}{ Groups } & \multicolumn{4}{|c|}{ Multiple comparisons } \\
\hline & C & 0 & M & $p$ value & C vs 0 & C vs M & O vs $M$ \\
\hline Hyperemia & & & & $0.001^{\mathrm{a}}$ & & & \\
\hline Negative & $0(0.0)$ & $3(42.9)$ & $0(0.0)$ & & & & \\
\hline Low & $1(14.3)$ & $4(57.1)$ & $7(100.0)$ & & & & \\
\hline Moderate & 3 (42.9) & $0(0.0)$ & $0(0.0)$ & & & & \\
\hline High & $3(42.9)$ & $0(0.0)$ & $0(0.0)$ & & & & \\
\hline Mean value (SD) & $2.29(0.76)$ & $0.57(0.54)$ & $1.00(0.0)$ & $<0.001^{b}$ & $<0.001^{c}$ & $<0.001^{c}$ & $0.151^{c}$ \\
\hline Median value (min-max) & $2(1-3)$ & $1(0-1)$ & 1 & $0.001^{d}$ & $0.003^{e}$ & $0.003^{e}$ & $0.060^{e}$ \\
\hline Hemorrhagic infiltration & & & & 0.002 & & & \\
\hline Negative & $0(0.0)$ & $7(100.0)$ & $5(71.4)$ & & & & \\
\hline Low & 3 (42.9) & $0(0.0)$ & $2(28.6)$ & & & & \\
\hline Moderate & $4(57.1)$ & $0(0.0)$ & $0(0.0)$ & & & & \\
\hline High & $0(0.0)$ & $0(0.0)$ & $0(0.0)$ & & & & \\
\hline Mean value (SD) & $1.57(0.54)$ & $0.0(0.0)$ & 0.29 (0.49) & $<0.001$ & $<0.001$ & $<0.001$ & 0.217 \\
\hline Median value (min-max) & $2(1-2)$ & 0 & $0(0-1)$ & $<0.001$ & 0.001 & 0.004 & 0.141 \\
\hline Average Histological Score, mean (SD) & $3.86(1.22)$ & $0.57(0.54)$ & $1.29(0.49)$ & $<0.001$ & $<0.001$ & $<0.001$ & 0.119 \\
\hline Number of Goblet cells & $50.54(3.56)$ & $52.71(2.84)$ & $52.51(2.83)$ & 0.370 & 0.206 & 0.249 & 0.905 \\
\hline
\end{tabular}

Peroxidase levels were not significantly different in groups $\mathrm{C}$ and $\mathrm{O}(\mathrm{p}=0.666$; LSD test).

No statistically significant difference of TEAC levels was observed between the three groups $(\mathrm{p}=0.277$;ANOVA)

Analysis of variance revealed statistically significant differences in SOD levels between the three groups $(\mathrm{p}<0.001)$. Post-hoc analysis using LSD test revealed that, compared to group C, SOD levels were by $33.3 \%$ lower in Group $\mathrm{O}(\mathrm{p}<0.001)$ and $44.2 \%$, lower in group $\mathrm{M}(\mathrm{p}<0.001)$. Moreover, SOD levels were significantly lower in Group M compared to Group O, by $16.3 \%$, $(\mathrm{p}=0.047)$.
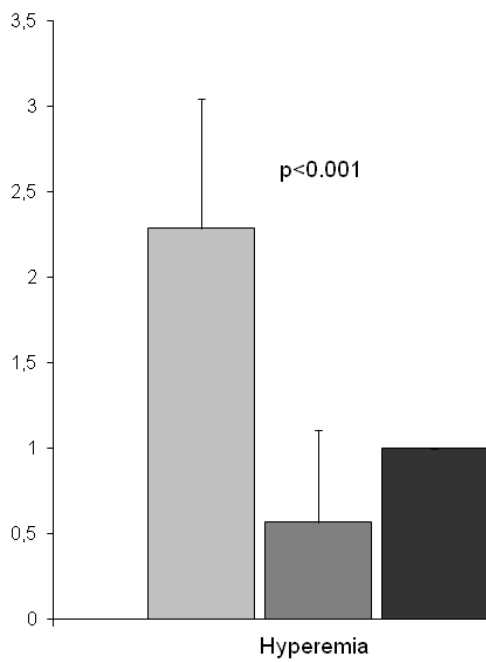

Regarding the anti-inflammatory properties, analysis of variance showed statistically significant differences in TNF- $\alpha$ and IL- $1 \beta$ levels between the three groups (Table 3).

TNF-a levels were $34.6 \%$ lower in Group M compared to Group C ( $\mathrm{p}=0.100)$ and significantly lower, by $45.7 \%$, compared to Group O $(\mathrm{p}=0.013)$.

Compared to Group C, lower IL- $1 \beta$ levels by $44.9 \%$, were observed in Group O ( $\mathrm{p}=0.001)$ and by $63.9 \%$, in Group M ( $<<0.001)$.

Moreover, there was a $34.6 \%$ lower level of IL-1 $\beta$ in Group M compared to Group O ( $\mathrm{p}=0.120$ ), but this difference did not reach the statistical significance.

\section{Fig. 4. Graphic of statistic comparison of colonic mucosal lesions in the three groups}



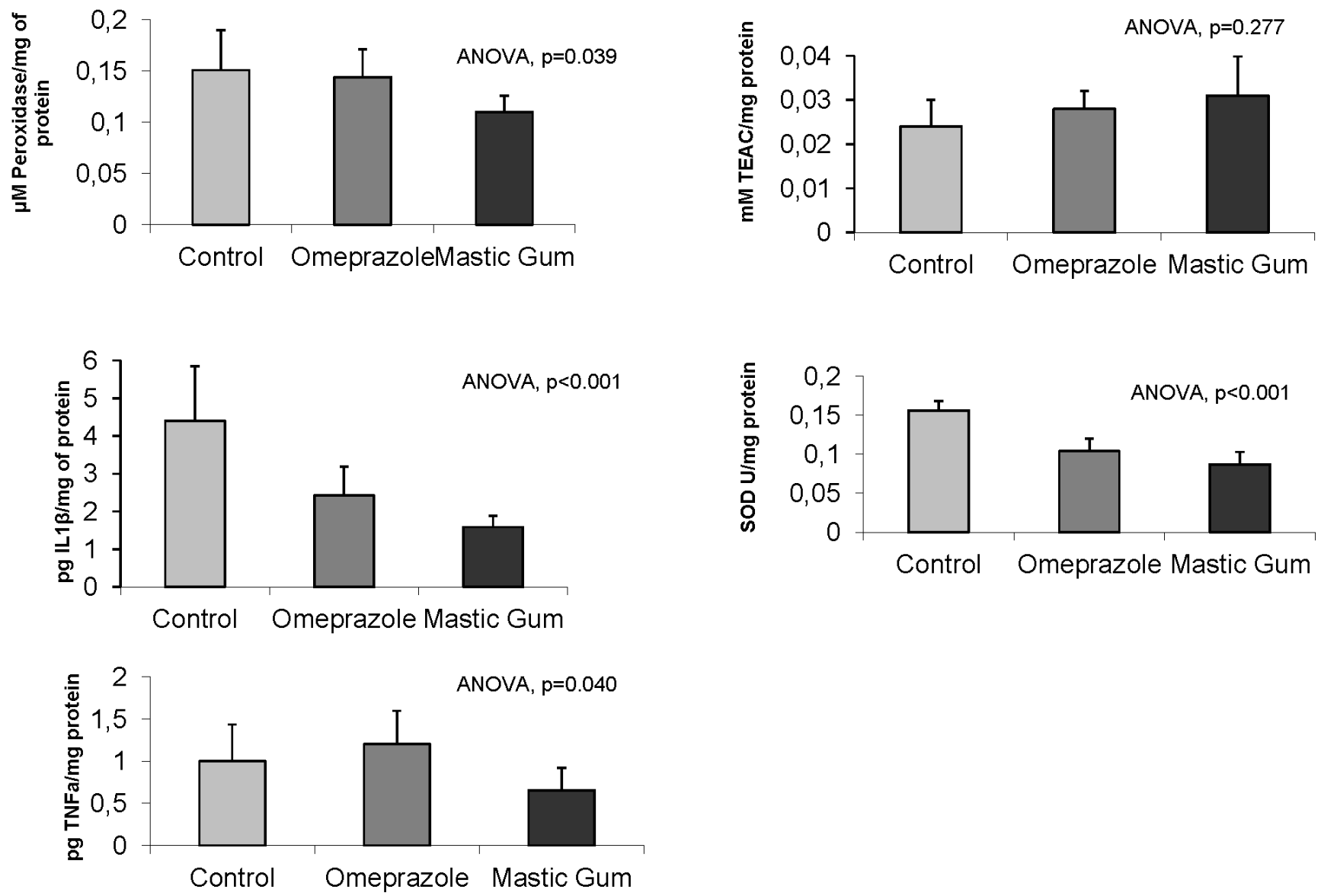

Fig. 5. Graphics of statistic comparison of antioxidant and anti- inflammatory markers between the three groups

\section{DIsCUSSION}

Numerous studies have documented that CRS induces gastric mucosal lesions as a result of oxidative damage caused by the significant generation of $-\mathrm{OH}[13,22$, 28-30].

In the present study, CMG was tested vs Omeprazole as a protection of the gastric and colonic mucosa against CRS induced by a mild severity protocol. The antioxidant and anti-inflammatory effects of the two agents were also evaluated.
Omeprazole is a selective and irreversible proton pump inhibitor, which suppresses stomach acid secretion by specific inhibition of the $\mathrm{H}^{+} / \mathrm{K}^{+}$-ATPase system found at the secretory surface of gastric parietal cells $[40,41]$. Biswas et al. (2003) have demonstrated that Omeprazole not only acts by decreasing gastric acid secretion [44].

It blocks the stress-induced increased generation of - $\mathrm{OH}$ and associated lipid peroxidation and protein oxidation, indicating that its antioxidant role plays a major part in preventing oxidative damage. Omeprazole also

Table 3. Serum antioxidant and anti-inflammatory markers in the three groups

\begin{tabular}{|c|c|c|c|c|c|c|c|}
\hline & \multicolumn{3}{|c|}{ Groups } & \multicolumn{4}{|c|}{ Multiple comparisons ${ }^{b}$} \\
\hline & C & $\mathbf{0}$ & M & p value a & C vs 0 & C vs $\mathrm{M}$ & O vs $\mathrm{M}$ \\
\hline$\mu \mathrm{M}$ Peroxidase/mg of protein & $0.151(0.039)$ & $0.144(0.027)$ & $0.110(0.016)$ & 0.039 & 0.666 & 0.018 & 0.043 \\
\hline $\mathrm{mM}$ TEAC/mg protein & $0.024(0.006)$ & $0.028(0.004)$ & $0.031(0.009)$ & 0.277 & 0.386 & 0.114 & 0.450 \\
\hline SOD U/mg protein & $0.156(0.012)$ & $0.104(0.016)$ & $0.087(0.016)$ & $<0.001$ & $<0.001$ & $<0.001$ & 0.047 \\
\hline pg TNFa/mg protein & $0.999(0.437)$ & $1.202(0.396)$ & $0.653(0.264)$ & 0.040 & 0.322 & 0.100 & 0.013 \\
\hline pg IL1 $\beta /$ mg of protein & $4.399(1.450)$ & $2.426(0.762)$ & $1.587(0.291)$ & $<0.001$ & 0.001 & $<0.001$ & 0.120 \\
\hline
\end{tabular}

a comparison between groups (one-way ANOVA); b pair-wise comparisons between groups (LSD test). C: Control O: Omeprazole M: Mastic Gum. TEAC: Trolox Equivalent antioxidant capacity, SOD: Superoxide dismutase. 
prevents stress-induced DNA fragmentation caused by .OH in vitro [44]. However, serious side effects associated with Omeprazole may include Clostridium difficile colitis, an increased risk of pneumonia or bone fractures. Moreover, acting as an inhibitor of the enzymes CYP2C19 and CYP3A4, Omeprazole may alter the absorption and plasma levels of anticoagulants, antidepressants, analgesics and some antibiotics [40, 41].

Consistent with previous experimental findings, histological changes of the gastric mucosa were observed in the herein study, such as hyperaemia, haemorrhagic infiltration, loss of EC, mucosal oedema and presence of IC in all three groups of animals, while true ulcers were not detected. The beneficial preventive effect of either Omeprazole or mastic gum compared to the control group was more pronounced for hyperaemia, haemorrhagic infiltration, loss of EC and oedema and almost non-existent for the presence of IC. However, no statistically significant effect was evident when comparing Omeprazole vs mastic gum for any of the five histological parameters.

These results suggest that CMG acts as efficiently as Omeprazole in the prevention of hyperaemia and haemorrhagic infiltration, oedema and loss of EC in the gastric mucosa of rats caused by CRS.

Hyperaemia and haemorrhagic infiltration of the colonic mucosa were less pronounced after omeprazole or mastic gum administration compared to the control group, but not significantly different between the two agents. Based on the results, CMG prevents hyperaemia and haemorrhagic infiltration of the colonic mucosa caused by CRS.

Colonic mucin- containing cup cells that were quantitatively evaluated synthesise and secrete mucins, high molecular weight glycoproteins, which form a protective layer throughout the gastrointestinal (GI) tract. This layer acts as an effective barrier as shown by changes in mucins in inflammatory conditions of the GI tract, by the altered Goblet cell response in germfree animals, and by the enhanced mucus secretion seen in response to infections $[4,43]$. Quantitative and qualitative evaluation of the mucin was not evaluated in the present study.

Triantafyllou et al. (2011) have demonstrated that the anti-inflammatory activity of CMG is associated with its antioxidant activity [37]. Pro-inflammatory cytokines stimulate superoxide production by NADPH oxidases, thus providing feed-forward activation of inflammatory pathways. This superoxide production is inhibited by mastic gum. It is suggested by recent stud- ies that CMG acts as a peroxisome proliferator-activated receptor (PPAR) modulator [45]. PPARs are nuclear receptors that control cellular functions via transcription at the level of gene expression implicated in the pathways of metabolic syndrome, inflammation, atheromatosis, and cancer $[36,45]$.

In the present study, it was evident that the mastic gum affected the antioxidant enzymes peroxidase and SOD.

Administration of mastic gum significantly decreased the activity of both enzymes compared to the levels observed by the administration of Omeprazole. SOD, but not peroxidase, was also significantly reduced for Omeprazole compared to the control group.

Regarding the anti-inflammatory properties of CMG, the inhibition of pro-inflammatory cytokines such as prostaglandin E2 (PGE2) and nitric oxide (NO) is mainly attributed to the inhibition of iNOS and COX-2 protein expression by activated macrophages rather than the scavenging of $\mathrm{NO}$ [46].

Also, in studies on experimental colitis, CGM administration resulted in a significant reduction of inflammatory markers as TNF-a, IL-6, and ICAM-1 and downregulated NF-jB p65 [37, 38]. Papalois et al. [36] have demonstrated that Chios mastic as a whole rather than its acidic, neutral components or oleanolic acid, exerts an anti-inflammatory effect on the colonic mucosa in trinitrobenzene sulfonic acid (TNBS)-induced colitic in rats via NF-jB regulation.

Mastic gum, in the present study, demonstrated lower values for the pro-inflammatory cytokine TNF- $\alpha$ compared to Omeprazole and for IL-1 $\beta$ compared to the control group.

\section{- CONCLUSIONS}

Peptic mucosal damage induced by acute stress is a serious cause of morbidity and mortality in critically ill patients. In this study, a mild CRS protocol induced peptic mucosal alterations, which were decreased in animals pre-treated with either CMG or Omeprazole. Furthermore, CMG appeared to significantly reduce hyperaemia, haemorrhagic infiltration, oedema and loss of epithelial cells to the gastric mucosa as well as hyperaemia and haemorrhagic infiltration to the colonic mucosa. At the same time, its antioxidative and anti-inflammatory properties, tested by various markers, were found comparable to those of Omeprazole. 
Available online at: www.jccm.ro

It is concluded that Chios mastic resin is effective against CRS- induced peptic mucosal damage in rats.

The study showed promising results of the use of Chios mastic gum as a nutritional supplement and its incorporation in therapeutic research protocols in clinical practice.

\section{ACKNOWLEDGEMENTS}

This study was conducted at the Experimental Research Centre ELPEN Pharmaceuticals (ERCE grant number 1503, 2016), Athens, Greece, which also provided the research facilities for this project.

The authors would like to thank A. Zacharioudaki DVM, MLAS, E. Karampela, MSc, M. Karamperi, K. Tsarea, N. Psychalakis, A. Karaiskos, S. Gerakis, and E. Gerakis, staff members of the ERCE, for their important assistance during the experiments.

\section{REFERENCES}

1. Guth PH. Gastric blood flow in restraint stress. Dig Dis Sci. 1972;17: 807-13.

2. Abdel-Salam OM, El-Batran S. Pharmacological investigation of trimetazidine in models of inflammation, pain and gastric injury in rodents. Pharmacology. 2005;75:122-32

3. Castagliuolo I, Lamont J.T, Qi B. Acute stress causes mucin release from rat colon: role of corticotropin releasing factor and mast cells. Am J Physiol. 1996;271:884-92

4. Sutanto W, de Kloet ER. The use of various animal models in the study of stress and stress-related phenomena. Lab Anim. 1994;28:293-306

5. Takeuchi K, Amagase K. Roles of Cyclooxygenase, Prostaglandin E2 and EP Receptors in Mucosal Protection and Ulcer Healing in the Gastrointestinal Tract.Curr Pharm Des. 2018;24:200211.

6. Amagase K, Izumi N, Takahira Y, Wada T, Takeuchi $\mathrm{K}$. Importance of cyclooxygenase-1/prostacyclin in modulating gastric mucosal integrity under stress conditions. J Gastroenterol Hepatol. 2014;29 Suppl 4:3-10.

7. Takeuchi K, Kato S, Ogawa Y, Kanatsu K, Umeda M. Role of endogenous prostacyclin in gastric ulcerogenic and healing responses-a study using IP-receptor knockout mice. J Physiol. 2001;95:75-80.

8. Hayase M, Takeuchi K. Gastric acid secretion and lesion formation in rats under water-immersion stress. Dig Dis Sci.1986;31:166-71.

9. Kitagawa H, Fujiwara M, Osumi Y. Effects of water-immersion stress on gastric secretion and mucosal blood flow in rats. Gastroenterol.1979;77:298-302.

10. Zhong $\mathrm{C}$, Zhan $\mathrm{P}$, Tian $\mathrm{H}$, Wang $\mathrm{P}$, Lei F. Anti-ulcer activity of the
The Journal of Critical Care Medicine 2020;6(2) • 109

three different extracts of Ferula lehmannii Boiss leaf in rats. Pak J Pharm Sci. 2019 Nov;32(6):2625-32.

11. Adzu B, Balogun SO, Pavam E. Evaluation of the safety, gastroprotective activity and mechanism of action of standardised leaves infusion extract of Copaifera malmei Harms. J Ethnopharmacol. 2015;22:152-5.

12. Wang FY, Liu JM, Luo HH. Potential protective effects of Clostridium butyricum on experimental gastric ulcers in mice. World J Gastroenterol. 2015;8:340-51.

13. Batista LM, de Almeida AB, Lima GR. Gastroprotective effects (in rodents) of a flavonoid rich fraction obtained from Syngonanthus macrolepsis. J Pharm Pharmacol. 2014;66:44552.

14. Balogun SO, Damazo AS, de Oliveira Martins DT. Helicteres sacarolha A.gastroprotectiveand possiblemechanismofactions in experimental animals. J Ethnopharmacol. 2015;26:176-84.

15. Park CH, Son HU, Son M, Lee SH. Protective effect of Acer mono Max. sap on water immersion restraint stressinduced gastric ulceration. Exp Ther Med. 2011;2:843-8.

16. Takahashi T, Suzuki G, Nibuya M. Therapeutic effect of paroxetine on stress-induced gastric lesions in mice. Prog Neuropsychopharmacol Biol Psychiatry. 2012;36:39-43.

17. Zhao J, Harada N, Sobue K. Insulin-like growth factor-I reduces stress-induced gastric mucosal injury by inhibiting neutrophil activation in mice. Growth Horm IGF Res. 2009;19:136-45.

18. Shah PJ, Gandhi MS, Shah MB. Study of Mimusops elengi bark in experimental gastric ulcers. J Ethnopharmacol. 2003;89:30511.

19. Yesilada E, Gürbüz Í, Toker G. Anti-ulcerogenicactivityandisol ationofthe activeprinciplesfrom Sambucus ebulus L. leaves. J Ethnopharmacol. 2014;153:478-83.

20. Arun LB, Arunachalam AM, Arunachalam KD, Annamalai SK, Kumar KA. In vivo anti-ulcer, anti-stress, anti-allergic, and functional properties of gymnemic acid isolated from Gymnema sylvestre $\mathrm{R} \mathrm{Br}$. BMC Complement Altern Med. 2014;22:14-70.

21. Al-Said MS, Ageel AM, Parmar NS, Tariq M. Evaluation of mastic, a crude drug obtained from Pistacia lentiscus for gastric and duodenal anti-ulcer activity. J Ethnopharmacol. 1986;15:2718.

22. Katsanou E.S, Kyriakopoulou K, Emmanouil C, Fokialakis N, Skaltsounis A.L, Machera K. Modulation of CYP1A1 and CYP1A2 Hepatic Enzymes after Oral Administration of Chios Mastic Gum to Male Wistar Rats. PLOS One. 2014;9:e 100190.

23. Amang PA, Tan PV, Patamaken SA, Mefe MN. Cytoprotective and antioxidant effects of the methanol extract of Eremomastax speciosa in rats. Afr J Tradit Complement Altern Med. 2013;11:165-71.

24. Chul-Hong P, Hyung-U S, Minsik S. Protective effect of Acer mono Max. sap on water immersion restraint stress-induced gastric ulceration. Exper Ther Med. 2011;2: 843-8.

25. Takagi, K, Susumu O. A simple method for producing stress 
110 The Journal of Critical Care Medicine 2020;6(2) ulcers in the rat. Jpn J Pharmacol. 1968;18:9-18.

26. Guo S, Gao X, Cao JM et al. Gastric mucosal damage in water immersion stress: Mechanism and prevention with GHRP-6. World J Gastroenterol. 2012;18:3145-55.

27. Jia Y.T, Wei W, Ma B, et al. Activation of p38 MAPK by reactive oxygen species is essential in a rat model of stress-induced gastric mucosal injury. J Immunol. 2007;179:7808- 19.

28. Das D, Banerjee RK. Effect of stress on the antioxidant enzymes and gastric ulceration. Mol Cell Biochem. 1993;125:115-25.

29. Ye B, Zhou PY, Jia M. Absence of NF-kB subunit p50 ameliorates cold immobilisation stress-induced gastric ulcers. Biochem Biophys Res Commun. 2013;10:547-51.

30. Yoshikawa T, Naito Y, Kishi A, et al. Role of active oxygen, lipid peroxidation, and antioxidants in the pathogenesis of gastric mucosal injury induced by indomethacin in rats. Gut. 1993;34:732-7.

31. Van Hecke $T$, Basso V, De Smet S. Lipid and Protein Oxidation during in Vitro Gastrointestinal Digestion of Pork under Helicobacter pylori Gastritis Conditions.J Agric Food Chem. 2018;66:13000-10.

32. Bhattacharjee M, Bhattacharjee S, Gupta A, Banerjee RK. Critical role of an endogenous gastric peroxidase in controlling oxidative damage in $\mathrm{H}$. pylori-mediated and nonmediated gastric ulcer. Free Radic Biol Med. 2002;32:731-43.

33. Bandyopadhyay D, Biswas K, Bhattacharyya M, Reiter RJ, Banerjee RK. Gastric toxicity and mucosal ulceration induced by oxygen-derived reactive species: protection by melatonin. Curr Mol Med. 2001;1:501-13.

34. Andreadou I, Mitakou S, Paraschos S, Efentakis P, Magiatis $P$, Kaklamanis L, Halabalaki M, Skaltsounis L, Iliodromitis EK.Pistacia lentiscus L. reduces the infarct size in normal fed anesthetized rabbits and possess antiatheromatic and hypolipidemic activity in cholesterol fed rabbits. Phytomedicine. 2016, 15;23:1220-6

35. Kaliora AC, Kountouri AM: Chemopreventive activity of Mediterranean medicinal plants. In: Cancer PreventionFrom Mechanisms to Translational Benefits (Georgakilas A, ed.). InTech, Rijeka, Croatia, 2012, pp. 261-84.
Available online at: www.jccm.ro

36. Papalois A, Gioxari A, Kaliora AC. Chios mastic fractions in experimental colitis: implication of the nuclear factor $\mathrm{KB}$ pathway in cultured HT29 cells. J Med Food. 2012;15:974-83

37. Triantafyllou A, Bikineyeva A, Dikalova A et al. Antiinflammatory activity of Chios mastic gum is associated with inhibition of TNF-alpha induced oxidative stress. Nutrition J. 2011; 10:64-73.

38. Bebb JR, Bailey-Flitte N, Ala'Aldeen D, Atherton JC. Mastic gum has no effect on Helicobacter pylori load in vivo. J Antimicrob Chemother. 2003;52:522-3.

39. Loughlin M.F, Ala'Aldeen DA, Jenks PJ. Monotherapy with mastic does not eradicate $\mathrm{H}$. pylori infection from mice. J Antimicrob Chemother. 2003;51:367-71.

40. Bavishi C, Dupont HL. Systematic review: the use of proton pump inhibitors and increased susceptibility to enteric infection. Alim Pharmacol Ther. 2011 Dec;34(11-12):1269-81.

41. Shirasaka, Y; Sager, J. E.; Lutz, J. D.; Davis, C; Isoherranen, N. Inhibition of CYP2C19 and CYP3A4 by Omeprazole Metabolites and Their Contribution to Drug-Drug Interactions". Drug Metab. Dispos. 2013;41 (7): 1414-24.

42. Shimozawa N, Okajima K, Harada N. Contribution of sensory neurons to sex difference in the development of stress- induced gastric mucosal injury in mice. Gastroenterology. 2006;131:1826-34.

43. Kakagia DD, Tsaroucha A, Lambropoulou MA. Bowel of Flowers. Int J Surg Pathol. 2018:1066896918776800. doi: $10.1177 / 1066896918776800$.

44. Biswas K, Bandyopadhyay U, Chattopadhyay I, et al. A novel antioxidant and antiapoptotic role of Omeprazole to block gastric ulcer through scavenging of hydroxyl radical. J Biol Chem. 2003;278:10993-11001.

45. Georgiadis I, Karatzas T, Korou LM et al. Beneficial health effects of Chios Gum Mastic and peroxisome proliferatoractivated receptors: indications of common mechanisms. J Med Food. 2015;18:1-10.

46. Zhou L, Satoh K, Takahashi K, Watanabe S et al. Re-evaluation of anti-inflammatory activity of mastic using activated macrophages. In Vivo. 2009;23:583-90. 